\title{
Effect of triflumuron, a chitin synthesis inhibitor, on Aedes aegypti, Aedes albopictus and Culex quinquefasciatus under laboratory conditions
}

Thiago Affonso Belinato ${ }^{1,2}$, Ademir Jesus Martins ${ }^{1,2}$, José Bento Pereira Lima ${ }^{1,2}$ and Denise Valle ${ }^{1,2,3^{*}}$

\begin{abstract}
Background: Resistance to traditional insecticides represents a threat to the control of disease vectors. The insect growth regulators (IGR) are a potential alternative to control mosquitoes, including resistant populations. The chitin synthesis inhibitors (CSI) are IGRs, which interfere with the insect molting process and represent one major class of compounds against Aedes aegypti populations resistant to the larvicide organophosphate temephos. In the present study, we evaluated the efficacy of the CSI triflumuron on Culex quinquefasciatus, Aedes albopictus and against several Ae. aegypti field populations.
\end{abstract}

Methods: The efficacy of triflumuron, against $C x$. quinquefasciatus and Ae. albopictus was evaluated with laboratory strains through dose-response assays. Additionaly, this CSI was tested against seven Ae. aegypti field populations exhibiting distinct resistance levels to both temephos and the pyrethroid deltamethrin. Aedes aegypti populations were exposed to both a dose that inhibits $99 \%$ of the adult emergence of mosquitoes from the susceptible reference strain, Rockefeller, $\left(E_{9 g}=3.95 \mu \mathrm{g} / \mathrm{L}\right)$ and the diagnostic dose $(\mathrm{DD})$, corresponding to twice the $\mathrm{E}_{9 g}$.

Results: Our results indicate that triflumuron was effective in emergence inhibition (EI) of $C X$. quinquefasciatus $\left(\mathrm{El}_{50}=5.28 \mu \mathrm{g} / \mathrm{L} ; \mathrm{El}_{90}=12.47 \mu \mathrm{g} / \mathrm{L}\right.$ ) and Ae. albopictus $\left(E I_{50=} 1.59 \mu \mathrm{g} / \mathrm{L} ; \mathrm{El}_{90}=2.63 \mu \mathrm{g} / \mathrm{L}\right)$. Triflumuron was also effective against seven Ae. aegypti Brazilian populations resistant to both temephos and deltamethrin. Exposure of all the Ae. aegypti populations to the triflumuron $\mathrm{El}_{99}$ of the susceptible reference strain, Rockefeller, resulted in complete inhibition of adult emergence, suggesting no cross-resistance among traditional insecticides and this CSI. However, a positive correlation between temephos resistance and tolerance to triflumuron was observed.

Conclusion: The results suggest that triflumuron represents a potential tool for the control of disease vectors in public health. Nevertheless, they point to the need of constant monitoring of the susceptibility status of vector populations to CSIs.

Keywords: Triflumuron, Chitin synthesis inhibitors, Aedes albopictus, Culex quinquefasciatus, Aedes aegypti, Insecticide resistance

\section{Background}

Aedes aegypti, Aedes albopictus and Culex quinquefasciatus mosquitoes are widely distributed across the globe, mainly in tropical and subtropical regions [1]. The presence of these species is considered a public health problem,

\footnotetext{
*Correspondence: dvalle@ioc.fiocruz.br

'Laboratório de Fisiologia e Controle de Artrópodes Vetores, Instituto

Oswaldo Cruz-Fiocruz, Rio de Janeiro, RJ, Brazil

${ }^{2}$ Laboratório de Entomologia, Instituto de Biologia do Exército, Rio de

Janeiro, RJ, Brazil

Full list of author information is available at the end of the article
}

because they are involved in transmission of diseases, such as dengue, yellow fever and lymphatic filariasis [2-4].

In Latin America, the presence of these mosquitoes involves an additional risk, as they are also potential vectors of chikungunya and the West Nile virus, two arboviruses not yet reaching this continent [5-7].

Currently, organophosphates (OP) and pyrethroids (PY) still play an important role in vector control. However, their effectiveness has been hampered due to resistance [8-11]. In this sense, the use of new products is a crucial 
issue for the development of novel and rational control strategies $[12,13]$.

Compared to conventional insecticides, like OP and PY, insect growth regulators (IGRs) have distinct mechanisms of action and are more selective. Additionally, IGRs are safe for most non-target organisms, and are therefore considered as a promising alternative for insect control [12,14]. Chitin synthesis inhibitors (CSI) are IGRs that interfere with the insect moult. CSIs belong to the benzoyl urea family, a chemical group that has been extensively studied since its discovery in the 1970s [14]. CSI exposure results in deformities of the larval cuticle, which often become unable to survive to the next moult [15-17].

Although the CSI mechanism of action is unclear, there are several reports of its effectiveness against insects, especially in larvae [17-21]. However, since chitin is a molecule present in all life stages of insects, it is likely that a series of mosquito structures are affected as well. Wilson and Cryan, for example, verified that lufenuron affects Drosophila melanogaster eggs [22]. Moreover, it has been shown that exposure of larvae to partially lethal doses of CSIs causes a series of disabilities in both viability and reproduction of resulting adults [23-27].

The use of CSIs has been intensified against resistant mosquito populations, given their mechanism of action is distinct from neurotoxic insecticides traditionally employed. Thus, evaluation of the efficacy of CSI on Ae. aegypti field populations is essential for rational vector control measures. In addition, the study of CSI effects on other mosquitoes could be important for the development of integrated control strategies.

Under laboratory conditions, triflumuron is effective against Ae aegypti [20]. When larvae are exposed to a sublethal dose, the viability of resulting adults is affected [26]. In the present study, we evaluated the effect of this CSI in several Ae. aegypti Brazilian populations with distinct resistance levels to temephos and deltamethrin. Additionally, laboratory strains of $C x$. quinquefasciatus and Ae. albopictus, two other mosquitoes of medical importance, were exposed to several triflumuron doses, and the effects on adult emergence inhibition were evaluated.

\section{Methods}

\section{Mosquitoes}

Aedes aegypti populations were chosen according to their geographical location (Figure 1) and resistance levels to both temephos (OP) and deltamethrin (PY). Mosquitoes from the Rockefeller strain, an insecticide-susceptible reference lineage [28], were used as the experimental control.

Aedes albopictus and $C x$. quinquefasciatus strains were collected in Rio de Janeiro city and maintained in the laboratory for five and thirteen years, respectively.
However, these laboratory strains are not considered as insecticide susceptible reference strains.

In all cases, groups of 1,000 first instar larvae were reared in plastic basins $(33 \times 24 \times 8 \mathrm{~cm})$ containing $1 \mathrm{~L}$ of dechlorinated water and $1 \mathrm{~g}$ of cat food (Friskies ${ }^{\circledR}$, Purina, Camaquã/RS). Larvae from all species and populations were kept in a biological oxygen demand incubator (BOD) at $25 \pm 1^{\circ} \mathrm{C}$ until the third instar, when assays were performed.

\section{Insecticides}

The CSI trifumuron Starycide SC $0.48^{\circledR}$ (triflumuron $48 \%$ ) was provided by BayerCropscience. PESTANAL ${ }^{\circledR}$ analytical standard of temephos (97.5\%) and deltamethrin (99.7\%) were purchased from Sigma-Aldrich.

\section{Triflumuron bioassays}

Dose-response bioassays were performed to evaluate triflumuron efficacy against Ae. albopictus and $C x$. quinquefasciatus. Groups of 10 third instar larvae were exposed to $150 \mathrm{~mL}$ of triflumuron solution in transparent plastic cups. The concentrations ranged from $0.25 \mu \mathrm{g} / \mathrm{L}$ to $4.5 \mu \mathrm{g} / \mathrm{L}$ for Ae. albopictus and from $2.5 \mu \mathrm{g} / \mathrm{L}$ to $50 \mu \mathrm{g} / \mathrm{L}$ for $C x$. quinquefasciatus. One milliliter of a $2.5 \%(\mathrm{w} / \mathrm{v})$ solution of ground cat food (Friskies ${ }^{\circledR}$, Purina, Camaquã/RS) was supplied once. Eight replicates were used for each concentration. Daily, mortality at each stage was checked and the resulting adults were collected [20,26].

Aedes aegypti populations were submitted to two triflumuron doses: one corresponding to the dose inhibiting Rockefeller adult emergence at 99\% ( $\left.\mathrm{EI}_{99}\right)$ and the diagnostic dose (DD), defined as twice the $\mathrm{EI}_{99}$ [29]. For both concentrations, eight replicates were utilized under the same conditions, as described above. The $\mathrm{EI}_{99}$ and DD doses were $3.95 \mu \mathrm{g} / \mathrm{L}$ and $7.9 \mu \mathrm{g} / \mathrm{L}$, respectively [20]. For all populations, triflumuron assays were performed on the F2 generation; except Maceió (AL), with F3.

In contrast to the neurotoxic insecticides, triflumuron bioassays were monitored for several days, until all insects either died or reached adulthood. Adult emergence inhibition (EI) data were registered as soon as all control group specimens emerged [20]. The results were used to calculate the $\mathrm{EI}_{50}$ and $\mathrm{EI}_{90}$ values that correspond to the triflumuron doses necessary to inhibit adult development of 50 and $90 \%$ of the specimens, respectively.

\section{Temephos and deltamethrin bioassays to determine the resistance level of the Ae. aegypti populations}

Temephos resistance was evaluated through bioassays [29]. Groups of 20 third instar larvae were exposed to ten temephos concentrations in plastic cups with $100 \mathrm{ml}$ of solution. Four replicates were used for each temephos dose. 


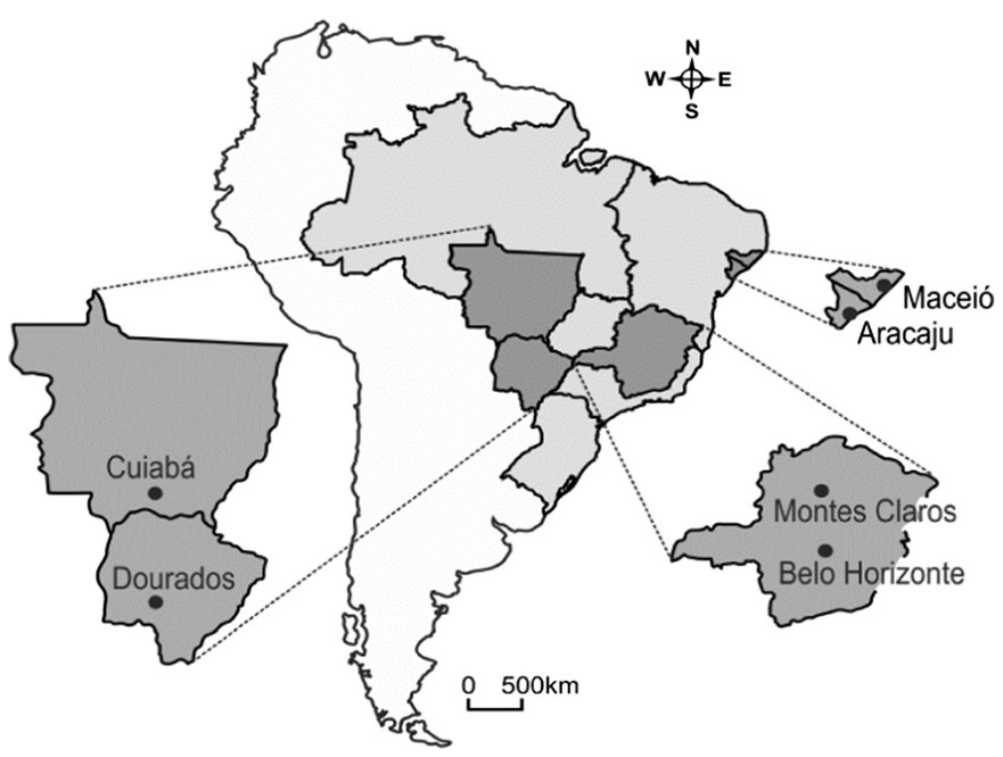

Figure 1 Geographic location of the Ae. aegypti populations adopted in triflumuron assays.

Deltamethrin resistance was investigated with adults as described by Da-Cunha et al. [9]. Each assay consisted of three $250 \mathrm{~mL}$ Wheaton bottles impregnated with the DD of this PY (5 $\mu \mathrm{g} /$ bottle), defined in this assay as the minimal amount of insecticide that kills all the specimens of a susceptible reference strain. One control bottle was impregnated only with acetone as solvent. Each bottle received 20 females, non-blood fed, one to three days old. Knock down (KD) was monitored at $15 \mathrm{~min}$ intervals up to 2 hours. Mosquitoes were then transferred to cages free of insecticide and mortality was registered again 24 hours later.

\section{Statistical analysis}

All the assays described here were repeated three times. The trifumuron effective doses $\mathrm{EI}_{50}$ and $\mathrm{EI}_{90}$ were calculated by probit analysis [30].

Since in Brazil the $R R_{95}$ values are used to guide recommendations of temephos application in the field, the resistance ratios (RR) of Ae. aegypti field populations to temephos were calculated with values of the lethal concentrations that kill 95\% of larvae $\left(\mathrm{LC}_{95}\right)$. RRs were obtained by dividing the LC of the field populations by the equivalent LC from the Rockefeller strain.

ANOVA was adopted to compare the rate of pupae mortality among Ae. aegypti populations. Pearson's correlation was employed to investigate the relationship between pupae mortality after exposure to triflumuron and resistance levels to temephos of Ae. aegypti field populations. The Graph-Pad Prism software version 5.0 for Windows was used to perform these analyzes (GraphPad Software, San Diego California USA, www. graphpad.com).

\section{Results}

Effect of triflumuron on Ae. albopictus

The effective $\mathrm{EI}_{50}$ and $\mathrm{EI}_{90}$ doses inhibiting emergence were $1.59 \mu \mathrm{g} / \mathrm{L}$ and $2.63 \mu \mathrm{g} / \mathrm{L}$, respectively (Table 1 ). No adult emergence occurred at concentrations above $3.0 \mu \mathrm{g} / \mathrm{L}$. Mortality in the control group, not exposed to the CSI, remained below 4\%. Figure 2 shows Ae. albopictus mortality at each stage and indicates the direct relationship between triflumuron concentration and the precocity of its effects. There were higher larval mortality rates at the highest triflumuron doses. Mortality at the pupal and adult stages was evident mainly in intermediate concentrations, respectively between $1.25 \mu \mathrm{g} / \mathrm{L}$ and $3.5 \mu \mathrm{g} / \mathrm{L}$ and at lower doses, up to $3.0 \mu \mathrm{g} / \mathrm{L}$.

\section{Effect of triflumuron on Cx. quinquefasciatus}

The $\mathrm{EI}_{50}$ and $\mathrm{EI}_{90}$ values for this species were $5.28 \mu \mathrm{g} / \mathrm{L}$ and $12.47 \mu \mathrm{g} / \mathrm{L}$, respectively (Table 1 ). No viable adults were recovered above $15.0 \mu \mathrm{g} / \mathrm{L}$. Mortality in the control group was $4.7 \%$. Stage-specific mortality of $C x$. quinquefasciatus exhibited the same pattern observed for Ae. albopictus (Figure 3). Larval mortality was directly proportional to the doses employed, while pupal and adult mortality rates were higher at intermediate and lower doses, respectively.

\section{Effect of triflumuron, deltamethrin and temephos on Ae. aegypti field populations}

Effective doses of triflumuron for Ae. aegypti remained between 1.5-1.8 fold below the equivalent ones for Ae. albopictus (Table 1). Triflumuron was effective against all field populations evaluated, regardeless of their resistance levels to deltamethrin and temephos (Table 2). In 
Table 1 Effective doses (ug/L) of triflumuron against the different mosquitoe species evaluated

\begin{tabular}{lccc}
\hline & Ae. aegypti* & Ae. albopictus & Cx. quinquefasciatus \\
\hline El50 & $0.86(0.80-0.93)$ & $1.59(1.54-1.63)$ & $5.28(4.15-6.72)$ \\
El90 & $1.80(1.51-2.17)$ & $2.63(2.42-2.64)$ & $12.47(8.85-17.60)$ \\
El99 & $3.95(2.46-4.49)$ & $3.95(3.35-4.68)$ & $25.11(12.9-48.86)$
\end{tabular}

Values between parentheses represent the confidence intervals $95 \%(95 \% \mathrm{Cl})$. *Martins et al. [20].

all cases, including the Rockefeller strain, mortality in the control groups remained below 3\%. Approximately ten days after the initiation of the experiments, all larvae from the control groups, reared without triflumuron, had already reached adulthood. In the same period, up to $86.3 \%$ and $99.2 \%$ of specimens were dead in the experimental groups exposed to the triflumuron $\mathrm{EI}_{99}$ and DD, respectively. Both concentrations completely blocked adult emergence in all populations evaluated (Table 2). However, a small proportion of larvae remained alive for a long period of time. When populations were exposed to $\mathrm{EI}_{99}$, total mortality resulted between 18 (Rockefeller strain) and 24 days (Montes Claros/MG) after the beginning of the bioassays. For DD, total mortality ranged between 17 and 52 days, in Maceió and Montes Claros samples, respectively.

Figure 4 shows the stage-specific mortality of several Ae. aegypti populations exposed to triflumuron $\mathrm{EI}_{99}$. In this figure, populations are arranged according to their temephos resistance levels, in growth order. The rate of dead pupae in the municipality of Aracaju/SE, the population exhibiting the highest temephos resistance level, was significantly higher than that in other populations (ANOVA; $\mathrm{p}<0.05$ ). Significant differences were also evidenced in the rate of pupal mortality of Maceió/AL and Montes Claros/MG when compared to the Rockefeller strain (ANOVA; $\mathrm{p}<0.05$ ). Except for Cuiabá/MT and Dourados North/MS, all these populations exhibited high temephos resistance levels, as shown in Table 2 $\left(\mathrm{RR}_{95}>10.0\right)$.

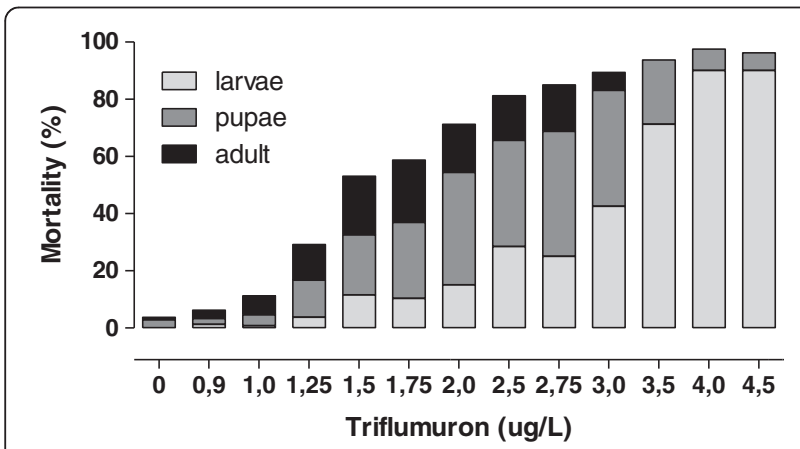

Figure 2 Stage-specific mortality of Ae. albopictus exposed to different triflumuron concentrations.

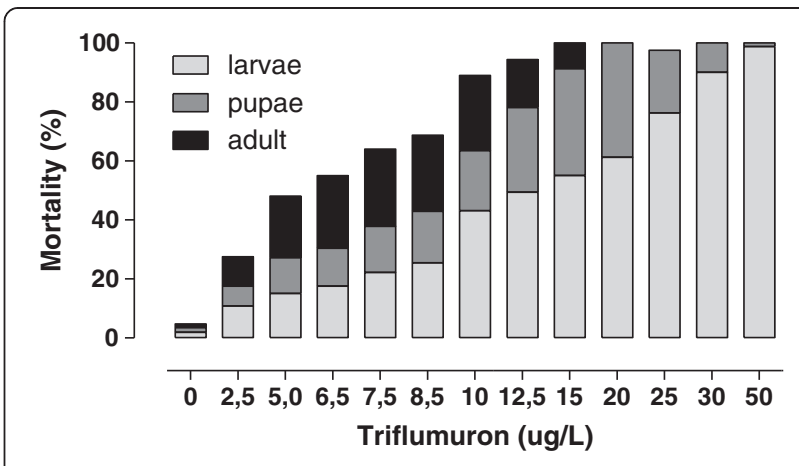

Figure 3 Stage-specific mortality of $C x$. quinquefasciatus exposed to different triflumuron concentrations.

In general, when field populations were exposed to triflumuron $\mathrm{EI}_{99}$, a positive correlation between temephos resistance levels and the rate of pupal mortality was found $\left(r^{2}=0,7293 ; p<0,05\right)$. The same correlation was not observed when populations were exposed to DD. In contrast to temephos resistance evaluation, deltamethrin bioassays were limited to exposure to a single dose of the pyrethroid. According to this bioassay, all Ae. aegypti populations were classified as resistant to deltamethrin (Table 2). Nevertheless, there was never a correlation between deltamethrin mortality rates and pupae mortality in Ae aegypti populations exposed to $\mathrm{EI}_{99}$ and DD of triflumuron noted.

\section{Discussion}

Insecticide resistance is a growing problem that hampers mosquito control in different regions around the world. Nowadays, resistance to the main neurotoxic insecticide classes has spread among several populations of insect disease vectors. Therefore, biological control methods and the use of chemicals with distinct mechanisms of action have been increasingly employed. The utilization of chitin synthesis inhibitors, for example, represents one major current strategy for mosquito chemical control. For this reason, evaluation of the efficacy of such compounds on mosquito field populations is essential. This work deals with the CSI triflumuron efficacy against Ae. aegypti field populations, as well as laboratory colonies of Ae. albopictus and Cx. quinquefasciatus.

Our results indicate that triflumuron, under laboratory conditions, is effective against the three mosquito species evaluated. As expected, this compound induced adult emergence inhibition of Ae. albopictus and Cx. quinquefasciatus in a dose-dependent way. Previous results of our group have proven that this compound acts in a similar way on Ae. aegypti [20]. Novaluron, another CSI, has the same effect on this mosquito [17]. Moreover, other IGRs, such as methoprene (juvenile hormone mimic) and halofenozide (ecdysone agonist), exhibited similar results [33,34]. 
Table 2 Temephos and deltamethrin resistance levels in Ae. aegypti field populations

\begin{tabular}{lcccc}
\hline Locality/Strain & $\begin{array}{c}\text { Temephos } \\
\mathbf{R R}_{\mathbf{9 5}}^{\mathbf{a}}\end{array}$ & $\begin{array}{c}\text { Deltamethrin } \\
\text { \% mort DD }\end{array}$ & \multicolumn{2}{c}{ Triflumuron,\% mort } \\
\cline { 3 - 5 } Rockefeller & 1.0 & 100.0 & 100.0 & 100.0 \\
Aracaju/SE & 19.3 & 88.1 & 100.0 & 100.0 \\
Maceió/AL & 10.3 & 62.9 & 100.0 & 100.0 \\
Belo Horizonte/MG & 5.4 & 74.5 & 100.0 & 100.0 \\
Montes Claros/MG & 13.6 & 62.7 & 100.0 & 100.0 \\
Dourados (North)/MS & 4.3 & 87.9 & 100.0 & 100.0 \\
Dourados-(South)/MS & 7.1 & 61.9 & 100.0 & 100.0 \\
Cuiabá/MT & 4.0 & 89.2 & 100.0 & 100.0 \\
\hline
\end{tabular}

${ }^{a}$ In Brazil, localities with Ae. aegypti populations exhibiting temephos $\mathrm{RR}_{95}$ higher than 3.0 are subjected to insecticide substitution [31]. Note that RR is based on dose-response assays, distinct from the remaining qualitative tests depicted in this table.

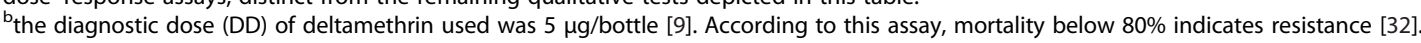

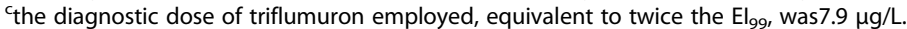

Several authors have observed that high doses of CSIs caused more pronounced mortality of mosquito larvae, relative to pupae and adults $[17,18,35]$. These results corroborate the data presented here, performed with triflumuron, and confirm the direct relationship between CSI concentrations and the precocity of their effects.

Triflumuron $\mathrm{EI}_{50}$ and $\mathrm{EI}_{90}$ against $C x$. quinquefasciatus (Table 1) were 3.3 and 4.7 times higher than the corresponding values in Ae. albopictus. In this latter species, in turn, triflumuron effective doses were around 1.5 1.8 times higher than in Ae. aegypti $\left(\mathrm{EI}_{50}\right.$ and $\mathrm{EI}_{90}$ of $0.86 \mu \mathrm{g} / \mathrm{L}$ and $1.8 \mu \mathrm{g} / \mathrm{L}$, respectively) [20]. The triflumuron doses recommended by the manufacturer for Aedes and Culex control are around $0.12 \mathrm{~g} / \mathrm{L}$ and $0.24 \mathrm{~g} / \mathrm{L}$, respectively [36], values much higher than the effective concentrations for these culicids.

Although there are some reports related to field simulated assays, to our knowledge this is the first evaluation of the triflumuron effect on Ae. albopictus under laboratory conditions. With respect to Cx. quinquefasciatus, our results slightly differ from other reports, which declared $\mathrm{EI}_{50}$ and $\mathrm{EI}_{90}$ values of 2.0 and $7.0 \mu \mathrm{g} / \mathrm{L}$, respectively $[37,38]$. Intraspecific variations of IGR effective doses among different laboratories are very common and are derived, in part, from the use of distinct protocols, as already discussed by Braga et al. [34]. Additionally, variations in effective doses among different CSIs for a given species, or among different insect species for a given CSI, have also been reported. Diflubenzuron, the first chitin synthesis inhibitor commercially available, has been used in the control of various insect species, especially in agriculture [14]. Concentrations of $0.5 \mu \mathrm{g} / \mathrm{L}$ of this compound resulted in $50 \%$ of $C x$. quinquefasciatus larval mortality [37]. Su et al. found for novaluron, another promising $\mathrm{CSI}, \mathrm{EI}_{50}$ and $\mathrm{EI}_{90}$ of respectively 0.16 and $0.60 \mu \mathrm{g} / \mathrm{L}$ for $C x$. quinquefasciatus [35]. Ali et al. studied the effect of diflubenzuron in Ae. albopictus and encoutered an $\mathrm{EI}_{50}$ of $0.45 \mu \mathrm{g} / \mathrm{L}$ for this species, the $\mathrm{EI}_{90}$ being $0.84 \mu \mathrm{g} / \mathrm{L}$ [39]. Hexaflumuron, another CSI, also proved very effective against Ae. albopictus, with the $\mathrm{EI}_{50}$ of $0.2 \mu \mathrm{g} / \mathrm{L}$ [40].

Although effective doses of triflumuron are higher when compared to other IGRs, Mian and Mulla emphasized the efficient activity of this CSI on mosquitoes, which is confirmed by its effectiveness in field trials [14]. Sulaiman et al. verified that $14.0 \mathrm{mg} / \mathrm{L}$ of triflumuron

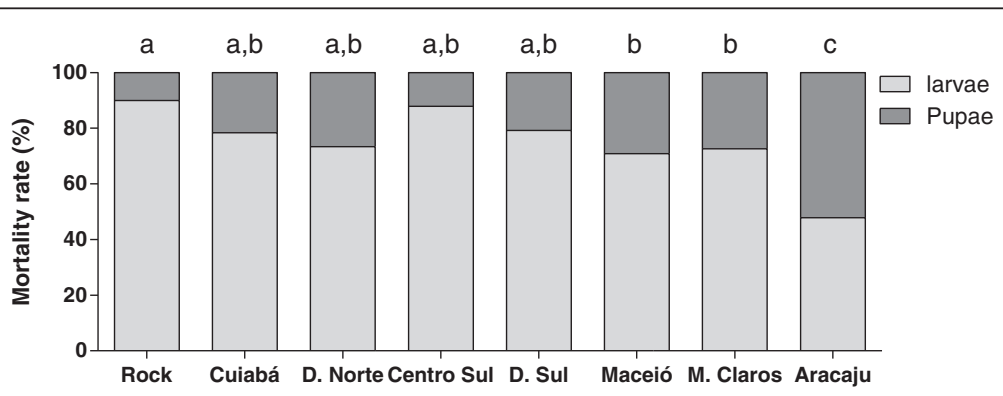

Figure 4 Stage-specific mortality induced by triflumuron of seven Ae. aegypti Brazilian populations exposed to El 99 . Populations are organized according to the resistance level to temephos. Distinct letters above columns indicate significant differences in pupae mortality rates $(p<0.05)$. 
inhibited Ae. albopictus emergence for 168 days under simulated field conditions [41]. Likewise, Batra et al. found that triflumuron was effective against $C x$. quinquefasciatus and Anopheles stephensi for seven weeks [42].

We also demonstrated that triflumuron is effective against Ae. aegypti field populations exhibiting different resistance levels to organophosphates and pyrethroids. In such cases, a small number of larvae always remained alive for many days, since the primary action of CSIs is not to induce mortality, but to interfere with development.

Aedes aegypti larval mortality was more pronounced when populations were treated with DD rather than $\mathrm{EI}_{99}$, confirming the direct relationship between CSI concentration and the precocity of its effects, likewise this has previously been shown for the Rockefeller strain [20]. However, total mortality occurred later in populations exposed to DD. Empirical observations during CSI bioassays indicate that in many cases, a small number of larvae remain alive for long periods, regardless of the dosage. However, these larvae never progress to adulthood. Probably, this occurs due to natural variations among specimens of field populations.

The absence of adult emergence when field populations are exposed to triflumuron, repudiates crossresistance between this CSI and neurotoxic inseticides. However, a positive correlation between pupal mortality rates and the temephos resistance ratios was noted, suggesting a potential triflumuron tolerance in populations resistant to this OP. This triflumuron tolerance could derive from the increased activity of enzymes related to metabolic resistance, already detected in several Brazilian Ae. aegypti populations [10,34]. In contrast, correlation between deltamethrin mortality rates and triflumuron induced pupae mortality in these Ae. aegypti populations were not detected. This result may in part be associated with the qualitative nature of the PY bioassay, consisting of a single dose. Although there are few reports concerning IGR resistance, diflubenzuron tolerance associated with mixed-function oxidases was evidenced in blowflies [43]. In mosquitoes however, to date IGR resistance has not yet been directly associated with increased activity of detoxifying enzymes.

\section{Conclusions}

Our results indicate that triflumuron was effective against the three mosquito species evaluated. Furthermore, this CSI was also potent against seven Ae. aegypti populations resistant to the two major classes of insecticides currently adopted in vector control. IGRs generally offer low toxicity to mammals, including man. Therefore, triflumuron might be considered an alternative for mosquito control in urban areas, although it has not yet been approved for application in drinking water, which precludes its use against Ae. aegypti [44].
Competing interests

The authors declare that they have no competing interests.

\section{Authors' contributions}

TAB designed the study, carried out all experiments and drafted the manuscript. AJM and DV also participated with the study design and critically reviewed the manuscript. JBPL scrutinized the protocol for the study and contributed with the interpretation of results. All authors read and approved the final version of the manuscript.

We dedicate this paper to the memory of Alexandre A. Peixoto, an outstanding friend and scientist.

\section{Acknowledgements}

This work was supported by the Conselho Nacional de Desenvolvimento Científico e Tecnológico (CNPq), Fundação de Amparo à Pesquisa do Estado do Rio de Janeiro (Faperj), Fundação Oswaldo Cruz (FIOCRUZ, PAPES and PDTSP programmes) The authors are grateful to BayerCropscience for the triflumuron samples. English review and revision by Mitchell Raymond Lishon, native of Chicago Illinois, U.S.A. - U.C.L.A. 1969. We dedicate this paper to the memory of Alexandre A. Peixoto, an outstanding friend and scientist.

\section{Author details}

${ }^{1}$ Laboratório de Fisiologia e Controle de Artrópodes Vetores, Instituto Oswaldo Cruz-Fiocruz, Rio de Janeiro, RJ, Brazil. 'Laboratório de Entomologia, Instituto de Biologia do Exército, Rio de Janeiro, RJ, Brazil. ${ }^{3}$ Instituto Nacional de Ciência e Tecnologia em Entomologia Molecular, Instituto de Bioquímica Médica, Universidade Federal do Rio de Janeiro, Rio de Janeiro, RJ, Brazil.

Received: 9 January 2013 Accepted: 22 March 2013

Published: 4 April 2013

\section{References}

1. Juliano SA, Lounibos LP: Ecology of invasive mosquitoes: effects on resident species and on human health. Ecol Letters 2005, 8:558-574.

2. Ottesen EA, Ramachandran CP: Lymphatic filariasis infection and disease: control strategies. Parasitol Today 1995, 11:129-131.

3. Gardner CL, Ryman KD: Yellow Fever: A Reemerging Threat. Clin Lab Med 2010, 30:237-260

4. Guzman MG, Halstead SB, Artsob H, Buchy P, Farrar J, Gubler DJ, Hunsperger E, Kroeger A, Margolis HS, Martínez E, Nathan MB, Pelegrino JL, Simmons C, Yoksan S, Peeling RW: Dengue: a continuing global threat. Nat Rev Microbiol 2010, 8:S7-S16.

5. Blitvich BJ: Transmission dynamics and changing epidemiology of West Nile virus. Anim Health Res Rev 2008, 9:71-86.

6. Ching Ng L, Hapuarachchi HC: Tracing the path of chikungunya virusevolution and adaptation. Infect Genet Evol 2010, 10:876-885.

7. Dibo MR, Menezes RMT, Ghirardelli CP, Mendonça AL, Neto FC: The presence of culicidae species in medium-sized cities in the state of São Paulo, Brazil and the risk of West Nile fever and other arbovirus infection. Rev Soc Bras Med Trop 2011, 44:496-503.

8. Liu H, Cupp EW, Guo A, Liu N: Insecticide resistance in Alabama and Florida mosquito strains of Aedes albopictus. J Med Entomol 2004, 41:946-952.

9. Da-Cunha MP, Lima JBP, Brogdon WG, Moya GE, Valle D: Monitoring of resistance to the pyrethroid cypermetrin in Brazilian Aedes aegypti (Diptera: Culicidae) populations collected between 2001 and 2003. Mem Inst Oswaldo Cruz 2005, 100:441-444.

10. Montella IR, Martins-Jr AJ, Viana-Medeiros PF, Lima JBP, Braga IA, Valle D: Insecticide resistance mechanisms of Brazilian Aedes aegypti populations from 2001 to 2004. AmJTrop Med Hyg 2007, 77:467-477.

11. Martins AJ, Lima JBP, Peixoto AA, Valle D: Frequency of Val1016lle mutation in the voltage-gated sodium channel gene of Aedes aegypti Brazilian populations. Trop Med Int Health 2009, 14:1351-1355.

12. Graf JF: The role of insect growth regulators in arthropod control. Parasitol Today 1993, 9:471-474.

13. Zaim M, Guillet P: Alternative insecticides: an urgent need. Trends Parasitol 2002, 18:161-163.

14. Mian LS, Mulla MS: Biological and environmental dynamics of insect growth regulators (IGRs) as used against Diptera of public health importance. Residue Rev 1982, 84:27-112. 
15. Reynolds SE: The cuticle, growth and moulting in insects: the essential background to the action of acylurea insecticides. Pest Science 1987, 20:131-146.

16. Tunaz $\mathrm{H}$, Uygun $\mathrm{N}$ : Insect growth regulators for insect pest control. Turk J Agric For 2004, 28:377-387.

17. Fontoura NG, Bellinato DF, Valle D, Lima JBP: The efficacy of a chitin synthesis inhibitor against field populations of organophosphate-resistant Aedes aegypti in Brazil. Mem Inst Oswaldo Cruz 2012, 107:387-395.

18. Mulla MS, Thavara U, Tawatsin A, Chompoosri J, Zaim M, Su T: Laboratory and field evaluation of novaluron, a new acylurea insect growth regulator, against Aedes aegypti (Diptera: Culicidae). J Vector Ecol 2003, 28:241-254

19. Ansari MA, Razdan RK, Sreehari U: Laboratory and field evaluation of himilin against mosquitoes. J Am Mosa Control Assoc 2005, 21:432-436.

20. Martins AJ, Belinato TA, Lima JBP, Valle D: Chitin synthesis inhibitor effect on Aedes aegypti populations susceptible and resistant to the organophosphate temephos. Pest Manag Sci 2008, 64:676-680.

21. Farnesi LC, Brito JM, Linss JG, Pelajo-Machado M, Valle D, Rezende GL: Physiological and morphological aspects of Aedes aegypti developing larvae: effects of the chitin synthesis inhibitor novaluron. PLoS One 2012, 7:e30363.

22. Wilson TG, Cryan JR: Lufenuron, a chitin-synthesis inhibitor, interrupts development of Drosophila melanogaster. J Exp Zool 1997, 278:37-44.

23. Vasuki V: Adult longevity of certain mosquito species after larval and pupal exposure to sublethal concentration of an insect growth regulator hexaflumuron. Southeast Asian J Trop Med Public Health 1992, 23:121-124.

24. Vasuki $V$ : The effects of sublethal doses of hexaflumuron on the feeding behavior of mosquitoes (Diptera: Culicidae). Bull Entomol Res 1992 82:535-538.

25. Vasuki V: Influence of IGR treatment on oviposition of three species of vector mosquitoes at sublethal concentrations. Southeast Asian J Trop Med Public Health 1999, 30:200-203.

26. Belinato TA, Martins AJ, Lima JBP, Lima-Camara TN, Peixoto AA, Valle D: Effect of the chitin synthesis inhibitor triflumuron on the development, viability and the reproduction of Aedes aegypti. Mem Inst Oswaldo Cruz 2009, 104:43-47.

27. Suman DS, Parashar BD, Prakash S: Effect of sublethal dose of diflubenzuron and azadirachtin on various life table attributes of Culex quinquefasciatus (Diptera: Culicidae). J Med Entomol 2010, 47:996-1002.

28. Kuno G: Early history of laboratory breeding of Aedes aegypti (Diptera: Culicidae) focusing on the origins and use of selected strains. J Med Entomol 2010, 47:957-971.

29. World Health Organization: Instructions for determining the susceptibility or resistance of mosquito larvae to insecticides (WHONBC/1981). Geneva: WHO; 1981.

30. Raymond M: Presentation d'une programme d'analyse logprobit pour microordinateur cahiers Orstrom. Sér Ent Med Parasitol 1985, 22:117-121.

31. Ministério da Saúde: Reunião técnica para discutir status de resistência de Aedes aegypti a inseticidas. Rio de Janeiro/Brazil: Coordenação Geral do Programa Nacional do Controle da Dengue. Relatório final, unpublished document, 27-29/2006 March, Rio de Janeiro/RJ.

32. Davidson G, Zahar AR: The practical implications of resistance of malaria vectors to insecticides. Bull WHO 1973, 49:475-483.

33. Boudjelida H, Bouaziz A, Soin T, Smagghe G, Soltani N: Effects of ecdysone agonist halofenozide against Culex pipiens. Pestic Biochem Physiol 2005, 83:115-123

34. Braga IA, Mello CB, Peixoto AA, Valle D: Evaluation of methoprene effect on Aedes aegypti (Diptera: Culicidae) development in laboratory conditions. Mem Inst Oswaldo Cruz 2005, 100:435-440.

35. Su T, Mulla MS, Zaim M: Laboratory and field evaluations of novaluron, a new insect growth regulator (IGR), against Culex mosquitoes. J Am Mosa Control Assoc 2033, 19:408-418.

36. Bayer Cropscience. [http://www.bayersaudeanimal.com.br/html/pdf/bula/ peixes/STARYCIDE_SC_480_BULA.pdf].

37. Mulla MS, Darwazeh $\overline{H A}$ : New insect growth regulators against flood and stagnant water mosquito - Effects on non target organisms. Mosq News 1979, 39:746.

38. Mulla MS: The future of insect growth regulators in vector control. J Am Mosa Control Assoc 1995, 11:269-273.
39. Ali A, Nayar JK, Rui-de X: Comparative toxicity of selected larvicides and insect growth regulators to a Florida laboratory population of Aedes albopictus. J Am Mosq Control Assoc 1995, 36:72-76.

40. Montada D, Rajavel AR, Vasuki V: Use of hexaflumuron, an insect growth regulator in the control of Aedes albopictus (Skuse). Southeast Asian J Trop Med Public Health 1994, 25:374-377.

41. Sulaiman S, Jeffery J, Sohadi RA: Residual efficacy of triflumuron against the dengue vector, Aedes albopictus (Skuse). Bull Soc Vector Ecol 1994, 1994(19):111-114.

42. Batra CP, Mittal PK, Adak T, Ansari MA: Efficacy of IGR compound Starycide 480 SC (Triflumuron) against mosquito larvae in clear and polluted water. J Vect Borne Dis 2005, 42:109-116.

43. Kotze AC, Sales N, Barchia IM: Diflubenzuron tolerance associated with monooxygenase activity in field strain larvae of the Australian sheep blowfly (Diptera: Calliphoridae). J Econ Entomol 1997, 90:15-20.

44. World Health Organization: Dengue: Guidelines for diagnosis, treatment, prevention and control. Geneva: WHO; 1999.

doi:10.1186/1756-3305-6-83

Cite this article as: Belinato et al:: Effect of triflumuron, a chitin synthesis inhibitor, on Aedes aegypti, Aedes albopictus and Culex quinquefasciatus under laboratory conditions. Parasites \& Vectors 2013 6:83.

\section{Submit your next manuscript to BioMed Central and take full advantage of:}

- Convenient online submission

- Thorough peer review

- No space constraints or color figure charges

- Immediate publication on acceptance

- Inclusion in PubMed, CAS, Scopus and Google Scholar

- Research which is freely available for redistribution 